\title{
Actualización. Indicaciones actuales para el uso de la hormona de crecimiento
} Growth hormone treatment update

\author{
Comité Nacional de Endocrinología de la Sociedad Argentina de Pediatría*
}

http:/ /dx.doi.org/10.5546/aap.2014.89

\section{RESUMEN}

La baja talla en la infancia es una causa frecuente de derivación al endocrinólogo infantil, y corresponde la mayoría de las veces a variantes normales del crecimiento.

Inicialmente la terapéutica con hormona de crecimiento humana estaba circunscripta a los niños que presentaban deficiencia de dicha hormona. A partir dela producción dela hormona recombinantehumana por ingeniería genética se pudo ampliar su uso a otras patologías.

Palabras clave: hormona de crecimiento recombinante humana.

\begin{abstract}
Short stature in children is a common cause for referral to pediatric endocrinologists, corresponding most times to normal variants of growth.

Initially growth hormone therapy was circumscribed to children presenting growth hormone deficiency. Since the production of recombinant human hormone its use had spread to other pathologies.

Key words: recombinant human growth hormone.
\end{abstract}

\section{INTRODUCCIÓN}

La baja talla en la infancia es una causa frecuente de derivación al endocrinólogo infantil, y corresponde la mayoría de las veces a variantes normales del crecimiento. ${ }^{1}$

Inicialmente la terapéutica con hormona de crecimiento (HC) estaba circunscripta a los niños que presentaban deficiencia de dicha hormona. A partir de la producción de la hormona recombinante humana por ingeniería genética se pudo ampliar su uso a otras patologías. ${ }^{2,3}$
La HC es un péptido compuesto por 191 aminoácidos secretado por la adenohipófisis. Su liberación está regulada por un factor liberador hipotalámico (GHRH) y un factor inhibidor (somatostatina). La deficiencia de HC produce talla y/o velocidad de crecimiento bajas y su exceso, gigantismo en la infancia y acromegalia en la adultez. Su principal acción se ejerce sobre el hueso y tejido conectivo estimulando el crecimiento, pero también tiene importantes funciones metabólicas; favorece la síntesis proteica y permite la disponibilidad de sustratos por medio de la lipólisis y la glucogenólisis. ${ }^{4}$

En esta actualización solo nos referiremos a las indicaciones que están aceptadas en nuestro país.

\section{OBJETIVOS}

- Orientar al pediatra en la evaluación general de los niños $y$ adolescentes con posible indicación de tratamiento con HC, y su derivación oportuna al especialista para llegar a un diagnóstico e indicación adecuada en forma conjunta.

- Actualizar las indicaciones más frecuentes y aceptadas en nuestro país.

- Informar al pediatra de cabecera sobre el monitoreo que requiere

Conflicto de intereses:

La Dra. Ana Keselman ha recibido honorarios por participar del consejo asesor científico de Novo Nordisk y Merck Serono.

*Dra. Viviana Pipman, Dr. Guillermo Alonso, Dra. María Eugenia Escobar, Dra. Titania Pasqualini, Dra. Ana Keselman, Dra. Elisabeth Boulgourdjian, Dra. Andrea Arcari, Dra. Sonia V. Bengolea, Dra. Silvia D'Amato. Coordinadoras: Dra. Ana Keselman y Dra. Titania Pasqualini. 
este tratamiento para asegurar la efectividad y la seguridad de la droga, tarea que será llevada a cabo por el especialista.

\section{Indicaciones actuales}

Actualmente en Argentina el uso de HC está indicado en la insuficiencia hipofisaria, síndrome de Turner, insuficiencia renal crónica, síndrome de Prader Willi y restricción del crecimiento intrauterino (Tabla 1).

El Ministerio de Salud cuenta con un programa de financiamiento para pacientes carentes de cobertura social y/o recursos, y establece las condiciones para aceptar al paciente dentro del mismo (www.infoleg.mecon.gov.ar).

\section{Insuficiencia hipofisaria}

La insuficiencia hipofisaria (IH) puede limitarse a deficiencia aislada de HC o estar asociada a deficiencias de otras trofinas hipofisarias. Puede ser idiopática u orgánica, congénita o adquirida. En este último caso puede deberse a traumatismos durante el nacimiento o posteriormente en la infancia, histiocitosis, linfoma o leucemia, tumores hipotálamohipofisarios o secuela de radioterapia. ${ }^{5-7}$

La sospecha diagnóstica se basa en datos auxológicos, talla baja para la talla parental y baja velocidad de crecimiento en ausencia de otra patología, y se apoya en antecedentes perinatales, ${ }^{8}$ evaluación clínica y estudios bioquímicos: IGF1 e IGFBP3 (factor de crecimiento insulino-símil y su proteína transportadora), pruebas dinámicas de la secreción de HC (arginina, clonidina, insulina, LDOPA $)^{9}$ y valoración de la maduración esquelética (Rx para edad ósea).

Los estudios por imágenes, especialmente la resonancia magnética nuclear cerebral (RMN) para evaluar las áreas selar y supraselar, permiten descartar una patología orgánica y ayudan al diagnóstico de las causas genéticas. Las características clínicas de la insuficiencia grave de $\mathrm{HC}$ en el recién nacido y en niños mayores se describen en las Tablas 2 y 3., 3,-8

El tratamiento consiste en la sustitución de la o las hormonas deficientes. En el caso de HC la administración es por vía subcutánea, en forma diaria y de preferencia nocturna y requiere de un monitoreo clínico y bioquímico, datos necesarios para la farmacovigilancia a corto y a largo plazo. ${ }^{10,11}$ Es posible alcanzar una talla adulta dentro del rango de la talla parental media. ${ }^{12}$

El Ministerio de Salud aprobó el uso de HC en los pacientes con deficiencia (Resolución $\mathrm{N}^{\mathrm{o}}$ 1346/07; Expediente n ${ }^{\circ}$ 1-2002-5086-06). Los criterios aprobados para iniciar el tratamiento por esta resolución están enumerados en la Tabla 4.

En los casos de pacientes con patologías

TABla 1. Año en que fue aprobado el tratamiento con hormona de crecimiento en niños sin deficiencia

\begin{tabular}{lccc}
\hline Aprobada & en EE.UU. & en Europa & en Argentina \\
\hline Síndrome de Turner $^{1}$ & 1996 & 1993 & 2007 \\
Insuficiencia renal crónica $^{2}$ & 1993 & 1995 & 2007 \\
Restricción de crecimiento intrauterino $^{3}$ & 2001 & 1995 (2003 Francia) & 2010 \\
Talla baja idiopática & 2003 & & no aceptada \\
\hline
\end{tabular}

1. Ministerio de Salud Pública. Resolución 1346/2007. Apruébanse las Normativas de Procedimientos para la solicitud de Hormona de Crecimiento en el marco de la Asistencia con Hormona de Crecimiento. Insuficiencia Hipofisaria o Síndrome de Turner.

2. Ministerio de Salud Pública. Resolución 1347/2007. Apruébanse las Normativas de Procedimientos para la solicitud de Hormona de Crecimiento para los pacientes que padecen Insuficiencia Renal Crónica.

3. Ministerio de Salud Pública. Resolución 2091/2010. Apruébanse las Normativas de Procedimientos para la solicitud de Hormona de Crecimiento para los pacientes que padecen Restricción de Crecimiento Intrauterino y/o Nacidos Pequeños para Edad Gestacional.

TABLA 2. Características clínicas/fenotipo de la insuficiencia grave de hormona de crecimiento en el recién nacido

- Hipoglucemia, convulsiones

- Hiperbilirrubinemia conjugada prolongada

- Hipotermia

- Micropene

- Defectos de la línea media: aumentan el riesgo de deficiencias aisladas o múltiples de hormonas hipofisarias 
TABLA 3. Características clínicas/fenotipo de la insuficiencia grave de hormona de crecimiento en los niños

- Retardo de crecimiento proporcionado y grave

- Acromicria

- Adiposidad troncal moderada

- Cara "de muñeca"

- Frente amplia

- Cabello fino

- Voz aflautada

- Retardo de la edad ósea

- Retardo en la dentición

orgánicas que producen deficiencia secundaria de HC se considerará más importante la velocidad de crecimiento que la talla para iniciar el tratamiento. En el caso de patología oncológica, se requiere por lo menos un año de seguimiento después de finalizado el tratamiento de la patología primaria y comprobar la ausencia de enfermedad. ${ }^{7}$

\section{Síndrome de Turner}

El síndrome de Turner (ST) es una entidad clínica descripta en 1938 por Henry Turner causada por una anomalía cromosómica con falta completa o parcial de un cromosoma X. Se define como la presencia de características clínicas sumadas a la ausencia completa o parcial del $2^{\circ}$ cromosoma sexual en línea pura o como parte de un mosaicismo cromosómico. Los cariotipos frecuentes con fenotipo similar son: 45, X; 46, XXr; 46, X, i (Xq); 45, X/46, XX. ${ }^{13}$

La incidencia es aproximadamente de 1/2500 a 3000 recién nacidas vivas. El diagnóstico puede realizarse en la etapa prenatal por estudio genético, en la recién nacida por la presencia de edemas de manos y/o pies asociados o no a cardiopatía, en la infancia por los estigmas físicos o en la adolescencia por alteraciones en la progresión de la pubertad. ${ }^{13}$ Las características clínicas / fenotipo del síndrome de Turner que deben alertar al pediatra para su derivación temprana se enumeran en la Tabla $5 .{ }^{14}$

El crecimiento de estas niñas está alterado, presentando disminución de la velocidad de crecimiento que a veces se hace notoria a partir de los 4 años de edad. La talla final promedio de una población de niñas argentinas con síndrome de Turner sin tratamiento fue de $137,9 \mathrm{~cm}$, o sea $20 \mathrm{~cm}$ menos que la talla media poblacional. ${ }^{15,16}$ Si bien no se conocen completamente las causas del retardo del crecimiento, la haploinsuficiencia del gen SHOX, ubicado en la región pseudoautosómica del Xp, Yp (p22.3) es una de las causas conocidas que explicaría en parte esta alteración del crecimiento.

TABLA 4. Criterios para iniciar el tratamiento con HC aprobados en nuestro país

\begin{tabular}{lcccc}
\hline & Edad (años) & Estatura (referencia nacional) & VC (Estándar británico) & Edad ósea (años) \\
\hline IH & & percentilo $<3$ o talla baja para padres & percentilo $<10$ & $\leq 13$ niñas $\leq 14$ niños \\
ST & $>4$ & percentilo $<3$ & percentilo $<10$ & $\leq 12$ \\
RCIU & $>5$ & $<2,5$ DS de la media & percentilo $<50$ & $\leq 11$ niñas $\leq 12$ niños \\
\hline
\end{tabular}

IH: Insuficiencia hipofisaria. ST: Síndrome de Turner. RCIU: Restricción de crecimiento intrauterino.

TABLA 5. Características clínicas/fenotipo del síndrome de Turner que deben alertar al pediatra ${ }^{14}$

- Cualquier niña con uno o más:

- Baja talla inexplicada

- Cuello alado

- Linfedema periférico

- Coartación de aorta

- Retraso puberal

- Cualquier niña con dos o más:

- Displasia ungueal

- Paladar arqueado y alto

- Cuarto metacarpiano corto

- Estrabismo

- Otros datos sugestivos:

- Retraso de aprendizaje no verbal, epicantus, ptosis palpebral, cúbito valgo, múltiples nevos, malformaciones renales (riñón en herradura, doble sistema excretor), aorta bicúspide (otras: anormalidades cardíacas izquierdas: estenosis aórtica, CIA, CIV, prolapso mitral), OMA recurrente. 
A pesar de que los niveles plasmáticos de la HC son normales, se ha demostrado que el tratamiento con HC produce una ganancia de talla final que varía entre 5 y $17 \mathrm{~cm} \cdot{ }^{17-19}$ Los mejores predictores de respuesta al tratamiento son la edad de inicio, la dosis de HC y la talla parental. La mayor ganancia de estatura se observa cuando el tratamiento se inicia alrededor de los 6,5 años. ${ }^{17,18}$ Por lo tanto, es muy importante que el pediatra esté alerta para que el diagnóstico y el tratamiento se realicen a edad temprana, lo que permitirá lograr una mejor talla y poder iniciar la terapéutica hormonal sustitutiva para inducir la pubertad a una edad similar a la de sus pares. ${ }^{13,17-20}$

Se demostró que los mayores determinantes de baja calidad de vida de estas pacientes son las malformaciones cardíacas $(12 \%)$ y las anormalidades en la audición (26\%); se observó también que los puntajes de percepción de salud eran menores en las pacientes cuya pubertad había sido inducida después de los 15 años. ${ }^{21}$

Los criterios para iniciar el tratamiento con $\mathrm{HC}$ en pacientes con Síndrome de Turner aprobados en nuestro país están enumerados en la Tabla 4.

\section{Insuficiencia renal crónica}

Los niños con insuficiencia renal crónica (IRC) presentan en forma frecuente restricción de crecimiento. Las causas de la baja talla son multifactoriales: anemia, acidosis, osteodistrofia renal, anomalías en el eje hipotálamo hipofisario, uso de medicaciones como corticoides por tiempo prolongado; el inicio temprano de la enfermedad es un factor contribuyente. ${ }^{22,23}$ Desde el año 1993 la Administración de Alimentos y Drogas (Food and Drug Administration - FDA) ha aprobado el uso del tratamiento con HC en esta patología. Independientemente de este tratamiento es importante intentar mejorar los factores que contribuyen al deterioro del crecimiento. ${ }^{23}$

Las condiciones para tratar con $\mathrm{HC}$ se enumeran en la Tabla 6. La respuesta al tratamiento es variable en relación con la etiología, el estado ácido base, la presencia de hiperparatiroidismo, la edad, la terapia sustitutiva renal y la duración de la IRC. Los inconvenientes a tener en cuenta son el deterioro de la función renal, el rechazo del trasplante, la proteinuria y la alteración del metabolismo glucídico. Un estudio realizado en Argentina demostró que la talla final de pacientes con trasplante renal tratados con $\mathrm{HC}$ fue significativamente mejor que la alcanzada por los controles sin tratamiento $(-1,88 \pm 1,14$ vs. $-3,48 \pm$ $1,19$ SDS, respectivamente, $p<0,05){ }^{24}$

\section{Restricción del crecimiento intrauterino}

Los niños que nacen con un bajo peso y/o talla para la edad gestacional es muy probable que hayan padecido una restricción en el crecimiento intrauterino (RCIU).

Las causas pueden ser muy variadas: placentarias, maternas, fetales o del medio ambiente. El 85\% de estos niños presentan crecimiento de recuperación en los primeros 2 a 3 años de vida (catch up). El 15\% que no lo presenta tiene riesgo de quedar bajo durante su infancia y 7-8\% finalizan con una talla adulta por debajo de lo normal. ${ }^{25}$ Las causas del retardo de crecimiento postnatal son multifactoriales. La ingesta calórica del niño

TABLA 6. Criterios para iniciar el tratamiento con HC aprobados en nuestro país para niños con insuficiencia renal crónica

\begin{tabular}{lcc}
\hline & Pre trasplante & Post trasplante \\
\hline Talla (percentilo) & $<3$ & $<3$ \\
Velocidad de crecimiento (percentilo) & $\leq 10$ & $\leq 10$ \\
Edad cronológica (años) & $>3$ & $>3$ \\
Edad ósea (años) & $<12 ;<13$ & $<12 ;<13$ \\
Filtrado glomerular $\left(\mathrm{ml} / \mathrm{min} / 1,73 \mathrm{~m}^{2}\right)$ & $>50$ & $>50$ \\
Hemoglobina $(\mathrm{mg} / \mathrm{dl})$ & $\geq 10$ & $\geq 10$ \\
Bicarbonato $(\mathrm{mmol} / \mathrm{L})$ & $\geq 20$ & $\geq 20$ \\
Hormona paratiroidea (veces sobre el valor normal) & $<4$ & $\geq 3$ \\
Albúmina $(\mathrm{g} / \mathrm{dl})$ & $\geq 3$ & $>78$ \\
Relación peso/talla (sin edemas) $(\%)$ & & 1 \\
Tiempo post trasplante $(\mathrm{año})$ & & $<0,25$ \\
Dosis de metilprednisona $(\mathrm{mg} / \mathrm{kg} / \mathrm{d})$ & & \\
\hline
\end{tabular}


debe estar garantizada. En la mayoría la secreción de HC es normal, pero en algunos casos se han descripto deficiencia o cuadros de resistencia a la misma. ${ }^{26}$

El tratamiento con $\mathrm{HC}$ está ampliamente aceptado en los niños sin recuperación de talla. En 161 niños tratados durante un promedio de 7,7 años la talla adulta media fue $<2,2 \mathrm{DE}$ $\mathrm{y}<1,1 \mathrm{DE}$ menor que la talla media parental. Los predictores de respuesta son la mejor talla al inicio, la respuesta durante el primer año de tratamiento, la duración del tratamiento y la talla parental. ${ }^{27}$

Es fundamental realizar durante el tratamiento el control del metabolismo de los hidratos de carbono, así como de los factores de crecimiento (IGF1 e IGFBP3).

Los criterios para iniciar el tratamiento con $\mathrm{HC}$ en pacientes con RCIU aprobados en nuestro país se enumeran en la Tabla 4.

\section{Síndrome de Prader Willi}

El síndrome de Prader Willi (SPW) es un desorden neurogenético complejo caracterizado por presentar anomalías del desarrollo psicomotor y baja talla. Fue descrito por primera vez en 1956 por Prader, Labhart y Willi quienes describieron pacientes hipotónicos con deficiencia mental, hipogonadismo, obesidad grave y diabetes. Su incidencia es de $1 / 10000$ a $1 / 25000$ recién nacidos vivos. ${ }^{28}$

La causa de este síndrome es, en 75\% de los pacientes, la deleción del alelo paterno de la zona 15q11-13; también puede ser causado por disomía materna uniparental $(15 \%)$ o anomalías del "imprinting". 29

Clínicamente estos niños presentan hipotonía y succión débil en el período neonatal, retardo de crecimiento en el primer año de vida y posteriormente hiperfagia compulsiva que los lleva a una obesidad mórbida. Además pueden presentar retraso madurativo, escoliosis, alteraciones en la termorregulación, apneas centrales y de tipo obstructivas. ${ }^{28,29}$ Desde el punto de vista endocrinológico, pueden presentar hipogonadismo, alteración en la secreción de HC, diabetes y menos frecuentemente hipotiroidismo. Su etiopatogenia estaría relacionada con una alteración a nivel de los núcleos hipotalámicos lo que explicaría muchos de los signos y síntomas que caracterizan esta patología. ${ }^{30}$

El tratamiento con HC está indicado no solo por el efecto sobre el crecimiento, sino por sus acciones metabólicas como el incremento de la masa magra, la disminución de la masa grasa (acción anabólica y lipolítica) y la mejoría de la hipotonía. Sin embargo está contraindicado en el caso de obesidad mórbida por el riesgo de muerte por apneas obstructivas. Es fundamental realizar controles polisomnográficos y consultas con Otorrinolaringología para la evaluación de las amígdalas y adenoides ya que su hipertrofia favorece la obstrucción de la vía aérea superior. ${ }^{31-34}$

\section{COMENTARIOS}

En IH, ST, IRC y RCIU el tratamiento con HC debe indicarse cuando la baja estatura del niño cumpla un papel central en su salud biopsicosocial y el aumento de la estatura inducido por el tratamiento participe, como factor importante, en una mejoría de su calidad de vida. En el recién nacido con $\mathrm{IH}$ el tratamiento puede ser una emergencia cuando se asocia con hipoglucemia y convulsiones. En los niños mayores los requerimientos de los padres, la opinión del pediatra y en algunos casos de los docentes deben tenerse en cuenta.

La efectividad del tratamiento con $\mathrm{HC}$ es mayor cuanto menor sea la edad de inicio y mayor el tiempo de tratamiento en etapa prepuberal, por esto el diagnóstico y la derivación a un endocrinólogo pediatra debe ser temprana.

En todos los casos es importante monitorear, no solamente la efectividad clínica, sino los efectos adversos que pueden aparecer. El tratamiento con $\mathrm{HC}$ es relativamente seguro, siendo los efectos colaterales más frecuentes dolor o hematoma en el lugar de aplicación y cefalea moderada. Los efectos adversos serios son raros e incluyen hipertensión intracraneal benigna, diabetes tipo II y desplazamiento de cabeza de fémur..$^{35}$

En todos los casos es importante controlar los niveles de los factores de crecimiento insulino símiles (IGF1 y su proteína de transporte IGFBP3). ${ }^{10,13,18}$

Se han realizado ensayos clínicos utilizando HC en otras patologías como síndrome de intestino corto $^{36}$ siendo los resultados inciertos y también en enfermedad fibroquística en donde solo se evaluó resultados a intervalos cortos. ${ }^{37}$

Existe un uso inapropiado de la $\mathrm{HC}$ en gimnasios o como terapia de rejuvenecimiento. Esta hormona se utilizó inicialmente solo para tratar pacientes con déficit de HC. Sin embargo el advenimiento de $\mathrm{HC}$ recombinante sintética desde la mitad de 1980 posibilitó su abuso como agente de "doping". El uso entre atletas de elite $\mathrm{y}$ en sujetos que asisten a gimnasios es bien 
conocido, aunque está prohibido por razones médicas y éticas. Es su efecto anabólico y en cierta medida su efecto lipolítico lo que más se valora. Sin embargo, últimamente, su efecto anabólico es cuestionado dado que no existe evidencia científica que en dosis suprafisiológica tenga un efecto adicional al logrado con entrenamiento y dieta. Aunque puede aumentar el volumen muscular, no aumenta la fuerza. Puede haber efecto sinérgico con otros anabólicos esteroides. Efectos adversos importantes son la retención de líquidos con edema de manos y pies y el síndrome del túnel carpiano. También pueden aparecer signos que caracterizan la acromegalia, hipertrofia de miocardio y mayor riesgo de cáncer. Existen datos que sugieren que puede tener un efecto beneficioso incrementando la síntesis de colágeno, lo que en un futuro podría aumentar su uso para prevenir o tratar rupturas de tendones y músculos. ${ }^{38,39}$

Es necesario aclarar que dentro del ámbito del Ministerio de Salud no se reconoce la cobertura del apoyo financiero solicitado en niños con baja estatura idiopática.

\section{BIBLIOGRAFÍA}

1. Allen DB, Cuttler L. Clinical practice. Short stature in childhood-challenges and choices. $N$ Engl $\mathrm{J} \mathrm{Med}$ 2013;368(13):1220-8.

2. Heinrich JJ. Terapéutica con hormona de crecimiento. Growth hormone therapy. Comentarios. Arch Argent Pediatr 2012;110(6):462-3.

3. Richmond E, Rogol AD. Current indications for growth hormone therapy for children and adolescents. Endocr Dev 2010;18:92-108.

4. Møller N, Jørgensen JO. Effects of growth hormone on glucose, lipid, and protein metabolism in human subjects. Endocr Rev 2009;30(2):152-77.

5. Mullis PE. Genetics of growth hormone deficiency. Endocrinol Metab Clin North Am 2007;36(1):17-36.

6. Taylor M, Couto-Silva AC, Adan L, Trivin C, et al. Hypothalamic-pituitary lesions in pediatric patients: endocrine symptoms often precede neuro-ophthalmic presenting symptoms. J Pediatr 2012;161(5):855-63.

7. Pasqualini T, Diez B, Domene H, Escobar ME, et al. Longterm endocrine sequelae after surgery, radiotherapy, and chemotherapy in children with medulloblastoma. Cancer 1987;59(4):801-6.

8. Braslavsky D, Keselman A, Galoppo M, Lezama C, et al. Neonatal cholestasis in congenital pituitary hormone deficiency and isolated hypocortisolism: characterization of liver dysfunction and follow-up. Arq Bras Endocrinol Metabol 2011;55(8):622-7.

9. Chaler EA, Rivarola MA, Guerci B, Ciaccio M, et al. Differences in serum GH cut-off values for pharmacological tests of GH secretion depend on the serum GH method. Clinical validation from the growth velocity score during the first year of treatment. Horm Res 2006;66(5):231-5.

10. Growth Hormone Research Society. Consensus guidelines for the diagnosis and treatment of growth hormone (GH) deficiency in childhood and adolescence: summary statement of the GH Research Society. J Clin Endocrinol Metab 2000;85(11):3990-3.

11. Takeda A, Cooper K, Bird A, Baxter L, et al. Recombinant human growth hormone for the treatment of growth disorders in children: a systematic review and economic evaluation. Health Technol Assess 2010;14(42):1-209.

12. Reiter EO,Price DA, Wilton P, Albertsson-Wikland K. Effect of grow th hormone $(\mathrm{GH})$ treatment on the near-final height of 1258 patients with idiopathic GH deficiency: analysis of a large international database. J Clin Endocrinol Metab 2006;91(6):2047-54.

13. Bondy CA. Turner Syndrome Study Group. Care of girls and women with Turner syndrome: a guideline of the Turner Syndrome Study Group. J Clin Endocrinol Metab 2007;92(1):10-25.

14. Lars Sävendahl MD, Marsha L, Davenport J. Delayed diagnoses of Turner's syndrome: Proposed guidelines for change. Pediatrics 2000;37(4):455-9.

15. Garcia Rudaz C, Martínez AS, Heinrich JJ, Lejarraga H, et al. Growth of Argentinian girls with Turner syndrome. Ann Hum Biol 1995;22(6):533-44.

16. Lejarraga H, Martínez A, García Rudaz C, Hauspie R, et al. Height velocity in Argentinean girls with Turner's syndrome. J Pediatr Endocrinol Metab 2001;14(7):883-91.

17. Baxter L, Bryant J, Cave CB, Milne R. Recombinant growth hormone for children and adolescents with Turner syndrome. Coch Datab Syst Rev 2007, Issue 1. Art. No.: CD003887. DOI: 10.1002/14651858.CD003887.pub2.

18. Van Pareren YK, de Muinck Keizer-Schrama SM, Stijnen $\mathrm{T}$, Sas TC, et al. Final height in girls with turner syndrome after long-term growth hormone treatment in three dosages and low dose estrogens. J Clin Endocrinol Metab 2003;88(3):1119-25.

19. Gutin L, Collier S, Bakalov VK, Bondy C. Trends in GH use in a Turner syndrome natural history study. Pediatr Endocrinol Rev 2012; 9(Suppl 2):725-7.

20. Michael B. Ranke A, Anders Lindberg B, Primus E, et al. Cutfield Maïthé Tauber David Dunger Towards Optimal Treatment with Growth Hormone in Short Children and Adolescents: Evidence and Theses. Horm Res Paediatr 2013;79:51-67.

21. Carel JC, Ecosse E, Bastie-Sigeac I, Cabrol S, et al. Quality of Life Determinants in Young Women with Turner's Syndrome after Growth Hormone Treatment: Results of the Stature Population-Based Cohort Study. J Clin Endocrinol Metab 2005;90(4):1992-7.

22. Tönshoff B, Kiepe D, Ciarmatori S. Growth hormone/ insulin-like growth factor system in children with chronic renal failure. Pediatr Nephrol 2005;20(3):279-89.

23. Fine RN, Martz K, Stablein D. What have 20 years of data from the North American Pediatric Renal Transplant Cooperative Study taught us about growth following renal transplantation in infants, children, and adolescents with end-stage renal disease? Pediatr Nephrol 2010;25(4):739-46.

24. Gil S, Vaiani E, Guercio G, Ciaccio M, et al. Effectiveness of rhGH treatment on final height of renal-transplant recipients in childhood. Pediatr Nephrol 2012;27(6):1005-9.

25. Albertsson-Wikland $K$, Karlberg J. Natural growth in children born SGA with and without catch up growth. Horm Res 2003;59(Suppl 1):129.

26. Albertsson-Wikland $K$, Boguszewski M, Karlberg J. Children born small-for-gestational age: postnatal growth and hormonal status. Horm Res 1998;49(Suppl 2):7-13.

27. Ranke MB, Lindberg A; KIGS International Board. Height at start, first-year growth response and cause of shortness at birth are major determinants of adult height outcomes of short children born small for gestational age and Silver- 
Russell syndrome treated with growth hormone: analysis of data from KIGS. Horm Res Paediatr 2010;74(4):259-66.

28. Char F. A photographic study: the natural history of PraderWilli syndrome. J Clin Dysmorphol 1984;2(1):2-4.

29. ButlerMG. Prader-WilliSyndrome: Obesity due to Genomic Imprinting. Curr Genomics 2011;12 (3): 204-15.

30. Bruni O, Verrillo E, Novelli L, Ferri R. Prader-Willi syndrome: sorting out the relationships between obesity, hypersomnia, and sleep apnea. Curr Opin Pulm Med 2010;16(6):568-73.

31. Allen DB, Carrel AL. Growth hormone therapy for PraderWilli syndrome: a critical appraisal. J Pediatr Endocrinol Metab 2004;17(Suppl 4):1297-306.

32. Coupaye M, Lorenzini F, Lloret-Linares C, Molinas C, et al. Growth hormone therapy for children and adolescents with Prader-Willi syndrome is associated with improved body composition and metabolic status in adulthood. JClin Endocrinol Metab 2013;98(2):E328-35.

33. Butler MG, Smith BK, LeeJ, Gibson C, et al. Effects of growth hormone treatment in adults with Prader-Willi syndrome. Growth Horm IGF Res 2013;23(3):81-7.

34. Deal CL, Tony M, Höybye C, Allen DB, et al. 2011 Growth
Hormone in Prader-Willi Syndrome Clinical Care Guidelines Workshop Participants. Growth Hormone Research Society workshop summary: consensus guidelines for recombinant human growth hormone therapy in PraderWilli syndrome. J Clin Endocrinol Metab 2013;98(6):E107287.

35. Reh CS, Geffner ME. Somatotropin in the treatment of growth hormone deficiency and Turner syndrome in pediatric patients: a review. Clin Pharmacol 2010;2:111-22.

36. Wales PW, Nasr A, de Silva N, Yamada J. Human growth hormone and glutamine for patients with short bowel syndrome. Cochrane Database Syst Rev 2010;(6):CD006321. doi: 10.1002/14651858.CD006321.pub2.

37. Hardin DS, Adams-Huet B, Brown D, Chatfield B, et al. Growth hormone treatment improves growth and clinical status in prepubertal children with cystic fibrosis: results of a multicenter randomized controlled trial. J Clin Endocrinol Metab 2006;91(12):4925-9.

38. Ehrnborg C, Rosén T. Physiological and pharmacological basis for the ergogenic effects of growth hormone in elite sports. Asian J Androl 2008;10(3):373-83.

39. Buzzini SRR. Uso de la hormona del crecimiento por los atletas jóvenes. Pediatr Clin N Am 2007;54:823-43. 